\title{
Multi-focus and Multi-window Techniques for Interactive Network Exploration
}

\author{
Priya Krishnan Sundararajan, Ole J. Mengshoel and Ted Selker \\ Carnegie Mellon University, Silicon Valley, CA, USA
}

\begin{abstract}
Networks analysts often need to compare nodes in different parts of a network. When zoomed to fit a computer screen, the detailed structure and node labels of even a moderately-sized network (say, with 500 nodes) can become invisible or difficult to read. Still, the coarse network structure typically remains visible, and helps orient an analyst's zooming, scrolling, and panning operations. These operations are very useful when studying details and reading node labels, but in the process of zooming in on one network region, an analyst may lose track of details elsewhere. To address such problems, we present in this paper multi-focus and multi-window techniques that improve interactive exploration of networks. Based on an analyst's selection of focus nodes, our techniques partition and selectively zoom in on network details, including node labels, close to the focus nodes. Detailed data associated with the zoomed-in nodes can thus be more easily accessed and inspected. The approach enables a user to simultaneously focus on and analyze multiple node neighborhoods while keeping the full network structure in view. We demonstrate our technique by showing how it supports interactive debugging of a Bayesian network model of an electrical power system. In addition, we show that it can simplify visual analysis of an electrical power network as well as a medical Bayesian network.
\end{abstract}

Keywords: Computer Graphics, Methodology and Techniques, Interaction techniques

\section{INTRODUCTION}

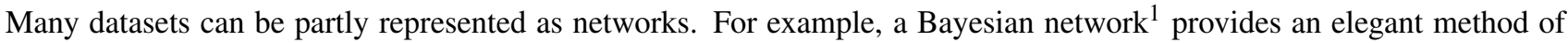
representing complex relationships among random variables in the form of networks. It is a directed acyclic graph model representing a set of variables, in the form of nodes, and their conditional dependencies, in the form of edges. Bayesian networks have become an important statistical machine learning tool and are being used effectively in uncertainty reasoning for many real world problems such as electrical power system diagnosis, ${ }^{2}$ medical diagnosis and image recognition.

As an example of an application, consider a Bayesian network for medical diagnosis. In this case, nodes may represent diseases and symptoms. The edges represent the causal relationship between diseases and their symptoms. The conditional probability table (CPT) table for each disease node reflect prior probabilities, while a symptom node CPT has probability values for the different combinations of states of its parent disease nodes. Given observed symptoms, also known as evidence, the network can be used to compute the posterior probability distribution for the presence of the diseases. Unlike other networks containing millions of nodes and edges, Bayesian networks found in most applications to date contain up to a few thousand nodes. In addition, the in- and out-degrees of Bayesian network nodes are quite small, typically a dozen or less, while some social networks nodes have thousands or millions of neighbors.

While software tools like Hugin ${ }^{3}$ and GeNIe/SMILE ${ }^{4}$ provide powerful visualization support for nodes, edges, and conditional probability tables (CPTs), many real-world Bayesian networks are becoming so large and complex that more advanced visualization and interaction techniques would be beneficial. Interactively investigating these networks, with hundreds or thousands of nodes and edges, can be quite challenging. ${ }^{1}$ Algorithmic Bayesian network analysis can be opaque, and existing visualization tools used for scrolling, zooming or panning the network do not elegantly combine network structure visualization with interactive exploration and understanding of node details. Even when merely moderately sized (say, with a few hundred nodes) Bayesian networks are zoomed to fit the screen, node labels may become unreadable. Consequently, an analyst may need to zoom, pan, and scroll in order to read node labels and thereby better

Further author information:

Priya: E-mail: priya.sundararajan@sv.cmu.edu

Ole: E-mail: ole.mengshoel@sv.cmu.edu

Ted: E-mail: ted.selker@sv.cmu.edu 
understand the joint role of different network nodes in Bayesian network computations. Unfortunately, in the process of zooming, panning, and scrolling, analysts may easily lose context. As an example, given a node of interest, it is hard to understand interactions with its children and parents if some of them are located far-off in the network layout. It is also difficult to keep values of the conditional probability tables (CPTs) in mind when studying and understanding zoomed-in details. Our multi-focus and multi-window visualization techniques enable a user to better compare and analyze internal details of nodes in different parts of a network while retaining network context.

The fisheye technique ${ }^{5}$ seeks to enable zooming in on details while retaining context; it was introduced to address the fundamental visualization problem of finding a specific address node in a address book structure of AT\&T employees. The fisheye technique maintains context and lets users study details, but allows focus on only one part of an information structure. In many cases, however, a user might need to compare multiple things in multiple parts of an information structure such as a network. Remembering the details of a previously studied zoomed node becomes a tedious memory taxing and error prone activity as the number of nodes for comparison increases. This is the limitation of traditional single fisheye techniques.

To overcome this limitation, as well as others, we formulate a number of design goals (DGs) that address our requirements associated with interactive exploration and analysis of networks, in particular Bayesian networks. The goals assume that zooming is being used. For the zooming algorithm, we integrate the previously stated goals ${ }^{6}$ and the suggestions from Bayesian experts. We hypothesized that the inherent complexity in Bayesian network representations could be overcome with visualization tools that focus on comparing parts of the network and their contents.

DG1 Multi-focus zooming: Multiple focus nodes selected in different parts of the network should be zoomed simultaneously, under user control, thus making their labels more readable regardless of their location in the network. The process of zooming in and zooming out should be animated, to avoid hard to follow abrupt layout changes.

DG2 Topology maintenance: The continuity of layout adjustment should not challenge the user's mental map of the structure of the network, that is the topology, and the proximity relations of the nodes should be maintained. ${ }^{7}$

DG3 Focus nodes selection: The user should be able to determine which nodes to zoom by studying the details associated with a subset of nodes. A similar set of nodes such as current nodes in an electrical network or disease nodes in an medical network, should be selectable by means of a search operation. A particular section of the network should also be selectable by means of a group selection operation.

DG4 Scoped zooming: The zooming should be restricted to a region of interest or a partition, around a focus node to avoid distorting the whole network layout. A partition algorithm should intuitively decide on the region of interest around the focus node for zooming. The set of nodes in the network should be partitioned accordingly. There is one partition per focus node. With no focus node, there is one partition for all the nodes. The partitions around the focus nodes is used to localize the zooming effect within that region.

DG5 Dynamic partitioning: The partitioning algorithm should dynamically adjust the partitions as the user interactively adds or removes focus nodes for scoped zooming.

DG6 No ghost regions: After zooming, discontinuities or 'ghost regions' between the partitions should not exist. ${ }^{8}$

DG7 Context visibility: We consider both local and global context. For local context visibility, the user should be able to control zooming to make the region surrounding the focus node more visible. For global context, the whole network should fit to the screen space and be visible during the user interactions. The user should still be able to control zooming the whole network.

DG8 Label zooming: The user should be able to make the node labels of focused nodes bigger and more easily readable. The degree of zooming decreases as we move away from the focus node.

DG9 Data exploration: Users should be able to simultaneously open multiple detail windows associated with the network nodes. Depending on the type of network, the data-level windows should show node details such as CPTs, time-series graphs or an individual's bio-data for comparison or data analysis. 


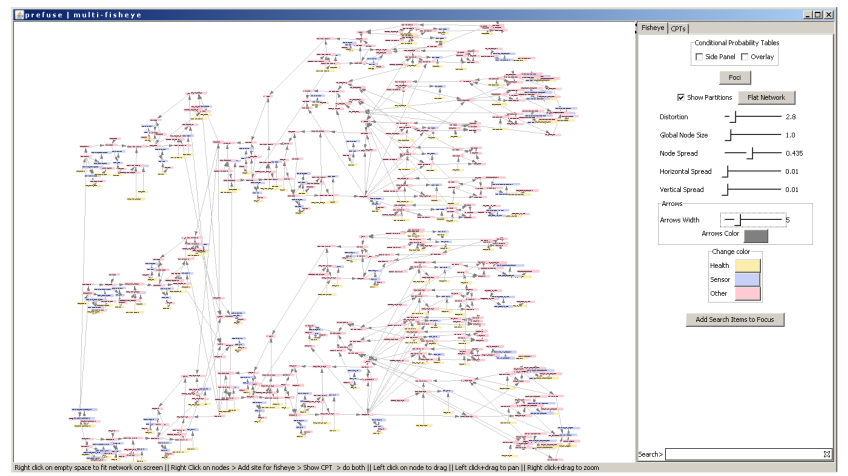

(a) Before fisheye zooming. Node labels are unreadable.

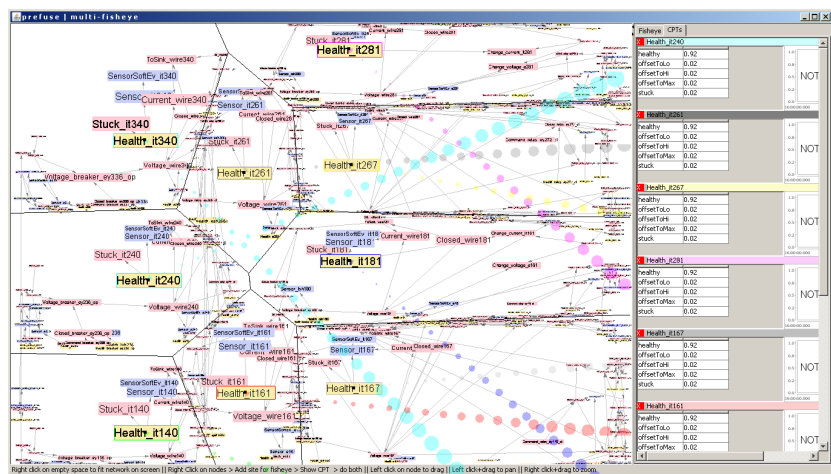

(b) After fisheye zooming. Node labels are readable.

Figure 1: Visualization of multi-variate probability distributions of ADAPT electrical Bayesian network. Side panel in Figure $1 \mathrm{~b}$ shows the multiple detail windows. The bubbles help the user to trace a detail window to its corresponding node in the network view.

Existing visualization techniques do not integrate all of the above design goals. We introduce in this paper a multi-focus technique to help analysts compare different parts of a network simultaneously while retaining network structural context, and especially aim to make the Bayesian analysis more effective in larger networks. Our novel approach satisfies the above goals and supports the visualization principles overview first, zoom and filter, then details-on-demand. ${ }^{9}$

Our software tool illustrated in Figure 1 has several GUI components. Our multi-focus technique starts with an initial layout in the network window as shown in Figure 1a. It allows analysts to select a set of focus nodes to zoom-in on multiple parts of a network. In order to minimize distortions while zooming, we localize a fisheye-like effect to a region surrounding a focus node. The distortion neighborhood is defined by partitioning the display into polygonal regions, using a Voronoi algorithm. ${ }^{10}$ All the nodes inside a polygonal region are closer to the focus node in that region than to the focus nodes in other regions. A localized fisheye zooming is applied to all partition regions, several such zoomed partitions can be created at the same time allowing users to zoom several parts of the network without losing structural context (see Figure 1b). The degree of distortion can be adjusted by using the slider in the control panel as shown in Figure 1. Our analysis and visualization is targeted on two tasks, the validation task and the diagnosis task. In the validation task, the analyst understands and checks the overall network by inspecting various nodes in different parts of the network. In the diagnosis task, the analyst focuses on the causal nodes that lead to a particular outcome, for an in-depth comparison and analysis. The analysts can also use the search operation in the control panel for selecting the nodes. There is also an option to show all available node details, in a dataview window.

The remainder of the paper is structured as follows: Section 2 provides an overview of previous related work, while the multi-focus algorithm is described in detail in Section 3. Application and expert evaluation are discussed in Section 4. Finally, Section 5 concludes the report with some hints for future research directions.

\section{RELATED WORK}

A number of existing techniques partially fulfill and motivate all the design goals (DG1 - DG9) outlined above. This section discusses how visualization, visual distortion, multi-focus and interactive visualization approaches might each improve on a user's ability to understand and solve problems in networks such as Bayesian networks.

\subsection{Network based fisheye techniques}

A number of focus+context display techniques have been introduced. ${ }^{11}$ A powerful and popular way to retain overview and detail is the fisheye approach. ${ }^{5}$ In a single-fisheye view, the entire node structure can be always visible. The user-selected node and its neighboring network nodes are magnified and distant nodes in terms of graph structure are demagnified. The fisheye techniques described by Sarkar and Brown ${ }^{12}$ use filtering and distortion, but support focus on a single item and distort the whole layout. Lamping et al. ${ }^{13}$ present an improved spatial distortion approach for the focus+context transformation using hyperbolic geometry. It places nodes around the root and provides smooth and continuous animation 
as users click or drag nodes to read the focus point of the layout. This approach also distorts the whole layout for each selection of focus point. In these techniques, some of our design goals are not satisfied, such as multi-focus zooming (DG1), topology maintenance (DG2), focus nodes selection (DG3), scoped zooming (DG4), dynamic partitioning (DG5) and data exploration (DG9). The design goals that are satisfied are continuity in layout (DG6), context visibility (DG7) and label zooming (DG8). Topology maintenance (DG2) is achieved in Furnas's fisheye approach ${ }^{5}$ but not in the hyperbolic approach. $^{13}$

Sarkar et al. ${ }^{14}$ also investigated a two focus approach with orthogonal and polygonal stretching, where the simple orthogonal distortion method maintains topological ordering of points (nodes maintain their left-of, above, etc. relationships), but the polygonal method does not. The zooming action is such that a user acts indirectly on the focus nodes through a 'rubber sheet.' This technique unfortunately produces violating the design goal, no ghost regions (DG6). Formella and Keller $^{15}$ distort network layout outside a polygonal area to make space for zooming all nodes inside the circumscribed polygon. This distortion mechanism does not scale to large networks as the user has to manually select the focus area by using a rectangular selection. Both these techniques do not support dynamic partitioning (DG5).

A topological fisheye method ${ }^{16}$ precomputes coarsened graphs and renders the level of detail from the combined graphs, depending on the distance from one or more foci. This system supports more than one focus (DG1). The drawback, however, is the computation involved in pre-computing the coarsened graphs, making it unsuitable for interactive exploration. Dynamic insets ${ }^{17}$ uses the connectivity of the graph to bring offscreen neighbours of on-screen nodes and their context into the viewport as insets. Unfortunately, the entire structure of network is not visible in this technique violating DG7. Feng et. al. recently introduced a multi-focus+context technique ${ }^{18}$ for generating spatiotemporal coherent time-varying graphs. This technique utilizes a triangle mesh to partition the graph nodes and leverages this underlying mesh for constrained multi-focus+context visualization. This was achieved through formulating an energy function for optimized deformation. This approach supports design goals such as the topology maintenance (DG2), continuity in layout (DG6) and context visibility (DG7). The above approaches do not support design goals such as focus nodes selection (DG3), scoped zooming (DG4), dynamic partitioning (DG5), label zooming (DG8) and data exploration (DG9). Other zoom algorithms ${ }^{19} 20$ provide more scalable multi-focus distortions, but without scoping of distortion, any focus change affects the entire network layout in these approaches. Also, the user has no direct control over the sizes of nodes aside from opening or closing them.

As described, previous techniques do not support one of our design goal, namely scoped zooming (DG4), as they distort the whole layout when rendering graphs at different levels. While the issue with distorting the whole layout is addressed by Reinhard et. al. in their improved fisheye zoom algorithm, ${ }^{6}$ it results in wasted screen space called 'ghost regions,' thus violating DG6.

\subsection{Tree based fisheye techniques}

Several previous systems demonstrate focus+context techniques for tree visualization, like SpaceTree, ${ }^{21}$ which uses extensive zooming animation to help users stay oriented within its focus+context tree presentation. Unfortunately, this technique does not support focus nodes selection (DG3), scoped zooming (DG4), dynamic partitioning (DG5), and creates ghost regions (DG6). The TreeJuxtaposer ${ }^{22}$ technique uses focus+context methods to support comparisons across hierarchical datasets. The technique also creates ghost regions (DG6). The reason is that this technique allows the user to do a rectangular selection which usually gives rise to 'ghost regions'. Also, these techniques require aggressive space constraint methods if the nodes of comparison are far apart in the tree structure.

Tu and Shen present 'balloon focus,' a multi-focus context technique for treemaps. Their user study confirms that users prefer the multi-focus treemaps to identify select players in a multi-year NBA dataset consisting of conferences, divisions, teams and player. ${ }^{23}$ While the treemaps provide good usage of the available space, network structure can be difficult to identify. ${ }^{24}$ So this approach does not retain the topology of the network (DG2). Bayesian networks are in general not trees, making tree visualizations limiting.

\subsection{Image based fisheye techniques}

Elmqvist et al. ${ }^{25}$ propose a new distortion technique that folds the intervening space to guarantee visibility of multiple focus regions. The folds themselves show contextual information and support unfolding and paging interactions. ${ }^{25} \mathrm{~A}$ drawback of this technique is the lack of user control over the scope of the focused regions. Non-linear magnification, ${ }^{26}$ pliable surfaces ${ }^{27}$ and compressed arc tangent graph algorithm, ${ }^{28}$ when applied to graphs, distort the labels within the zoomed areas. Such distortions make labels difficult to recognize or read (DG8). These techniques support multi-focus 


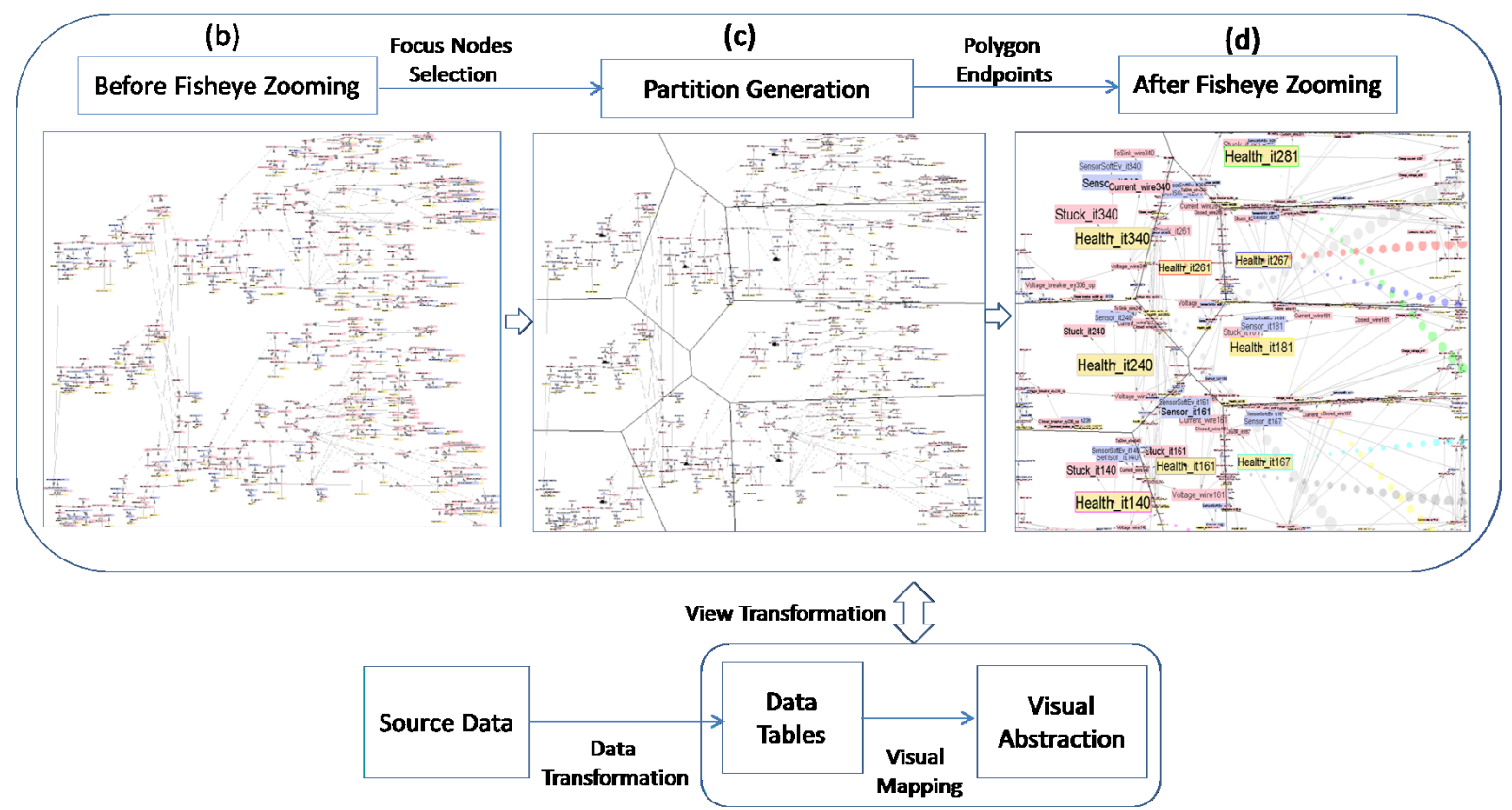

(a)

Figure 2: The diagram depicts the visulaization pipeline flow in our multi-focus algorithm. (a) The source data is loaded into Prefuse data tables and converted to visualizable attributes in the visual abstraction. View transformation takes place in three main steps: (b) before fisheye zooming; (c) partition generation; and (d) after fisheye zooming, also showing bubble anchors.

zooming (DG1), topology maintenance (DG2), dynamic partitioning (DG5), no ghost regions (DG6), and context visibility (DG8). But they do not support focus nodes selection (DG3), scoped zooming (DG4) (as the whole layout is distorted for each selection of focus point), label zooming (DG8) and data exploration (DG9).

\section{MULTI-FOCUS ZOOMING AND MULTI-WINDOW TECHNIQUES}

Our multi-focus zooming algorithm helps to retain the network structure, thus limiting distortion to preserve the user's mental map of the Bayesian network. The distortion algorithm is independent of the layout algorithm and is defined as a separate processing step on the layout of the graph. This allows for a modular organization of software ${ }^{29}$ and helped us to easily understand, modify and reuse existing code to suit our visualization. However, care must be taken for the fisheye distortion not to reduce readability of the display. To combat the negative aspect of distortion while giving an analyst control, we take the three steps shown in Figure 2:

- In the focus node selection step, the analyst selects a set of nodes for zooming. This can be done using a search operation over node labels or manually by selecting interesting nodes from the dataview window where all the detail windows of the nodes are displayed.

- After selection of the focus nodes, regions around the focus nodes are created by the partition generation step, which applies an incremental algorithm that maintains a set of partitions that varies over time by insertion or deletion. ${ }^{30}$ This is shown in Figure $2 \mathrm{c}$ which has nine focus nodes and polygonal partitions. This second step also ensures that all the nodes inside one partition are within that partition after distortion as shown in Figure 2d. This is done by measuring the maximum distance to move the node during distortion; the black lines as shown in Figure $4 \mathrm{~d}$ denotes the maximum distance to position the node so that it stays within the partition.

- The third step, fisheye zooming, distorts each partition as shown in Figure 2c. This results in zooming the focus nodes. For each focus node, the zooming gradually decreases as we approach the Voronoi edges of the partition. 
The above three steps repeat as the new focus nodes are selected and zoomed. The process of zooming in our approach therefore attempts to avoid the problems such as causing excessive distortions, excluding interactive rendering, excluding comparing multiple parts of a graph, excluding identifiers being readable or excluding simultaneous exploration of contents of nodes. Our technique is based on distorting the size and position of the label box based on its euclidean distances from the focus node. This helps to identify the focused node and its neighboring nodes.

Both Voronoi and rectangular partitioning approaches for a multi-focus technique have been proposed. ${ }^{31}$ We use the Voronoi partitioning approach ${ }^{32}$ as opposed to the traditional rectangular partitioning approach. ${ }^{31}$ This aids incremental partitioning (DG5) and continuous zooming layout (DG6) while preserving both structure and efficient use of space for arbitrary network structures. One benefit of the Voronoi approach is that it does not create ghost regions (DG6). After applying the fisheye technique locally inside each polygon, the nodes near the sides of all polygons are compressed, to preserve layout continuity. Previous work has, to our knowledge, not combined the Voronoi and the fisheye algorithm for multi-focus zooming.

In addition to multi-focus, our approach is multi-window. The recent GraphPrism [10] shows graph measures in stacked histograms and highlights nodes in a network based on selections in the histograms. We follow, and use multiple small windows to show more details such as the CPTs of the nodes. Having multiple windows with node detail information raises the question of maintaining a connection between a node and its details. We do this by means of 'lines of bubbles' (see Figure 5). Like bubbles connecting thoughts to a character in a cartoon, bubbles act as anchors and connect nodes to their details. Using these bubbles, the user can trace a detail window either in the side panel or floating, to its corresponding node in the network view. The bubbles and the title bar of the detail window have the same color to clearly show their connection. We use an improved version of bubbles previously used, ${ }^{33}$ replacing the solid row of large dots with a progressively enlarged row of hollow bubbles. By using hollow bubbles, the user can now more easily see the network underneath the bubbles, see Figure 7 for solid bubbles and Figure 5 for hollow bubbles. We experimented with different bubble color representations, solid versus hollow bubbles and different sizes of the bubbles. We found that hollow colored bubbles which progressively increase in size as it reaches the side panel are more effective.

The pseudo-code of the multi-focus algorithm is shown in Figure 3; we now discuss each of the three steps in more detail.

\subsection{Step 1: Selection of focus nodes}

Many current visualization techniques mainly address how to display the data while the user's primary concern, especially for large datasets, is what is selected for display. 5,34 We provide several options as discussed in the below Section 3.1 to help users to study the details associated with each node to find the truly interesting nodes, satisfying design goal, focus nodes selection (DG3).

1. The user can study the details of the nodes (detail windows) such as the time-series graphs or the CPTs as shown in the dataview window (DG9). If an interesting behavior is found, the detail window can be selected so that the corresponding node in the network window also gets selected.

2. In the network view, the detail windows can be viewed as a tooltip as the user hovers over a node. The detail window associated with a node can be anchored if the time-series graph or the CPT requires further inspection, see Figure 5.

3. A search operation can be used to select a set of nodes. This operation is useful, for example, when the user wants to study all the current nodes or the voltage-sensor nodes in an electrical network, see Figure 7.

4. Rectangular selection allows the user to select a group of nodes in a particular region of the network. After selection, the detail windows associated with those nodes can be opened and studied in the side panel, see Figure 5.

5. Users can select neighboring nodes in a graph. The selected nodes can be zoomed and studied, see Figure 8 and 10 . 


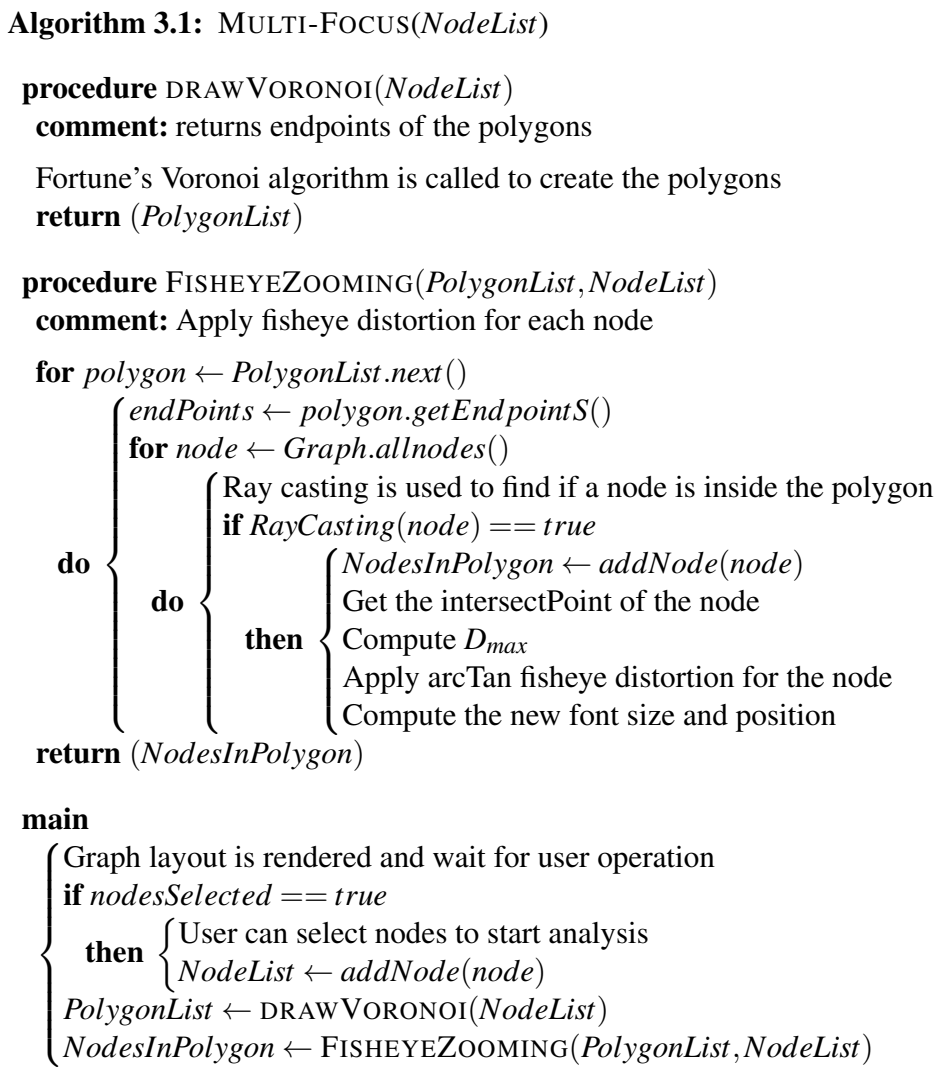

Figure 3: Pseudocode for multi-focus algorithm. The DrawVoronoi function takes the focus nodes (Nodelist) as inputs and outputs the endpoints of the polygons (PolygonList) for each focus node. The FisheyeZooming function takes the PolygonList and the NodeList as inputs and returns the distorted nodes (NodesInPolygon).

\subsection{Step 2: Partition Generation}

After selecting a set of focus nodes, a bounded region around each of the focus node should be automatically selected by the partitioning algorithm. The zooming algorithm is applied inside this region.

We experimented with a variety of rectangular partitioning approaches but found them causing discontinuities. ${ }^{31}$ The Voronoi algorithm ${ }^{30}$ can satisfy several design goals (DG4, DG5 and DG6); its works by dividing the display area into $n$ polygonal regions, given $n$ node selections. This algorithm is based on the principle that any node in the region will be nearer to the focus node in that region than to any focus nodes in neighboring regions.

When a node becomes a focus node, a partitioning algorithm ${ }^{30}$ is applied to that node and neigboring existing focus nodes to generate a new polygon, showing the incremental aspect of the algorithm. We apply the local fisheye to a bounded area by retrieving the corner co-ordinates of the region and updating the display accordingly. Each node in the graph is checked to see if it is present in the selected nodes' partitioned area using a ray casting technique. ${ }^{*}$ Only those nodes in the selected nodes' partitioned area undergoes the fisheye distortion.

\subsection{Step 3: Fisheye Zooming}

The analyst may want study the focus nodes in each partition. To do this, he may zoom-in on the focus nodes and the nearby nodes in the partitions. A local fisheye effect does this; the selected node is zoomed in and the size of the nearby nodes increases. We minimize the traditional fisheye effect that distorts the whole layout by localizing the fisheye effect within a partition. The user-selected node and its nearby nodes are magnified (DG7); the size of the nearby nodes decrease by the arctan of the distance from the focus as they reach the edge of the polygonal partition providing a continuous layout.

*http://www.ecse.rpi.edu/Homepages/wrf/Research/Short_Notes/pnpoly.html 
(a)

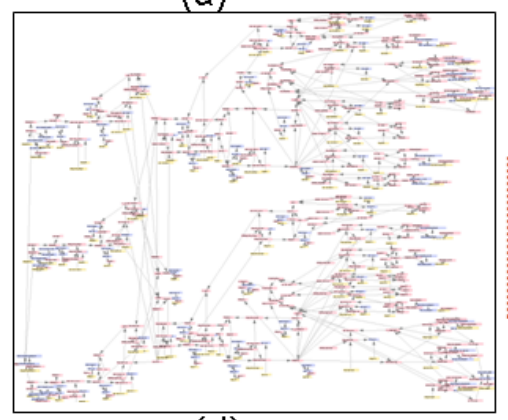

(d)

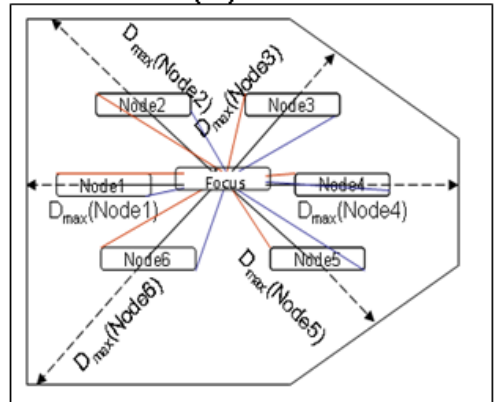

(b)

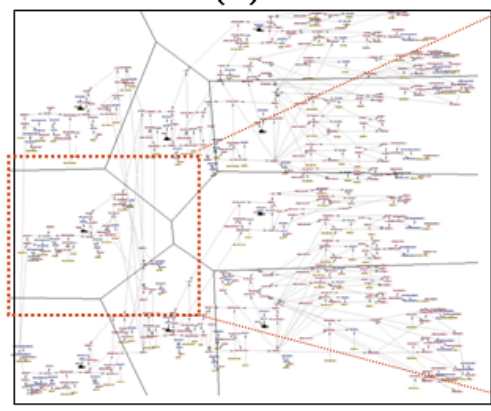

(e)

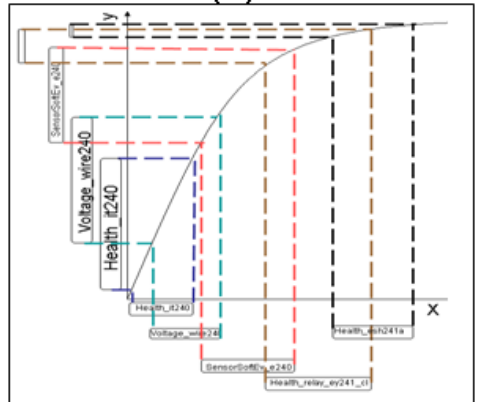

(c)

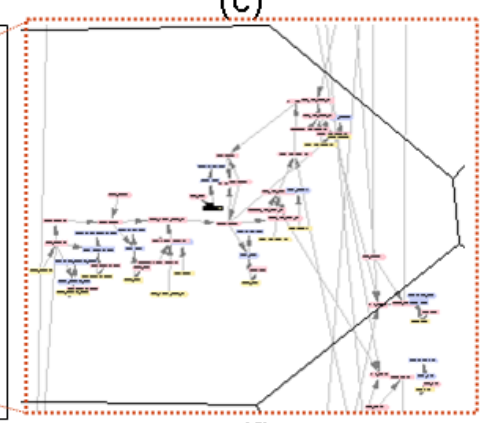

(f)

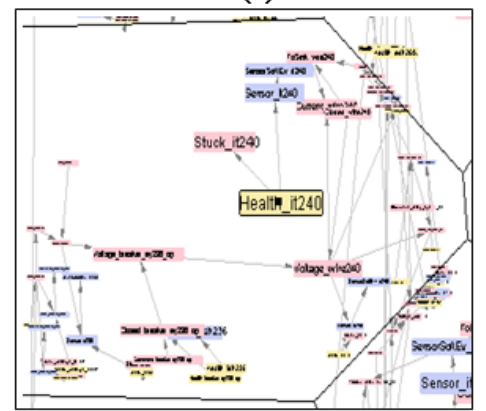

Figure 4: (a) A hard to read baseline network; (b) Partition lines have been drawn; (c) Viewing one partition; (d) Showing, in principle, how the maximum distance for each node label positions are computed (so that they do not move out of the polygon). Blue and red lines show the start and end distance of each node label rectangle from the focus; (e) arcTan distortion is applied for some node label rectangles based on the start(upper-left) and end(lower-right) coordinates; (f) Polygon shows focus nodes after distortion.

Each node in the network has start coordinates (upper-left corner) $\left(x_{s}, y_{s}\right)$, center coordinates $\left(x_{c}, y_{c}\right)$ and end coordinates (lower-right corner) $\left(x_{e}, y_{e}\right)$. Let $(x, y)$ denote either the start or the end coordinates of the node. The center coordinates of the focus node are denoted by $\left(x^{*}, y^{*}\right)$.The start and end distance of a node from the focus is denoted by $D_{s}$ and $D_{e}$. It is shown by the red and blue lines in Figure 4(d). The distance $D_{\max }$ is measured as the distance from the focus $\left(x^{*}, y^{*}\right)$ through the node's center $\left(x_{c}, y_{c}\right)$ to the point of intersection with the edge of the polygon as shown in Figure 4(d). These distance values are used to compute the transformed start $\left(x_{s}^{\prime}, y_{s}^{\prime}\right)$ and end $\left(x_{e}^{\prime}, y_{e}^{\prime}\right)$ co-ordinates of the node label box. The font size of the labels are then determined based on the difference between the transformed start and end co-ordinates in the $X$-dimension. The new position $\left(x_{c}^{\prime}, y_{c}^{\prime}\right)$ of the node is obtained by finding the center coordinates. The arctan fisheye distortion for start or end co-ordinates $(x, y)$ is done in the following steps:

Finding the distance of the node $(x, y)$ from the focus $\left(x^{*}, y^{*}\right)$ is done using:

$$
\begin{gathered}
d_{x}=x-x^{*} \\
d_{y}=y-y^{*} \\
D=\sqrt{\left(d_{x}\right)^{2}+\left(d_{y}\right)^{2}},
\end{gathered}
$$

where $D=D_{s}$ or $D=D_{e}$ and $(x, y)=\left(x_{s}, y_{s}\right)$ or $(x, y)=\left(x_{e}, y_{e}\right)$.

Conversion from Cartesian to Polar co-ordinates is done using:

$$
\theta=\arctan \left(\frac{d_{y}}{d_{x}}\right) .
$$

Normalizing the distance so that the node is not moved outside its Voronoi polygon:

$$
d_{\text {norm }}=D / D_{\max } .
$$

Calculation of radial distance $r$ and de-normalization is done using: 


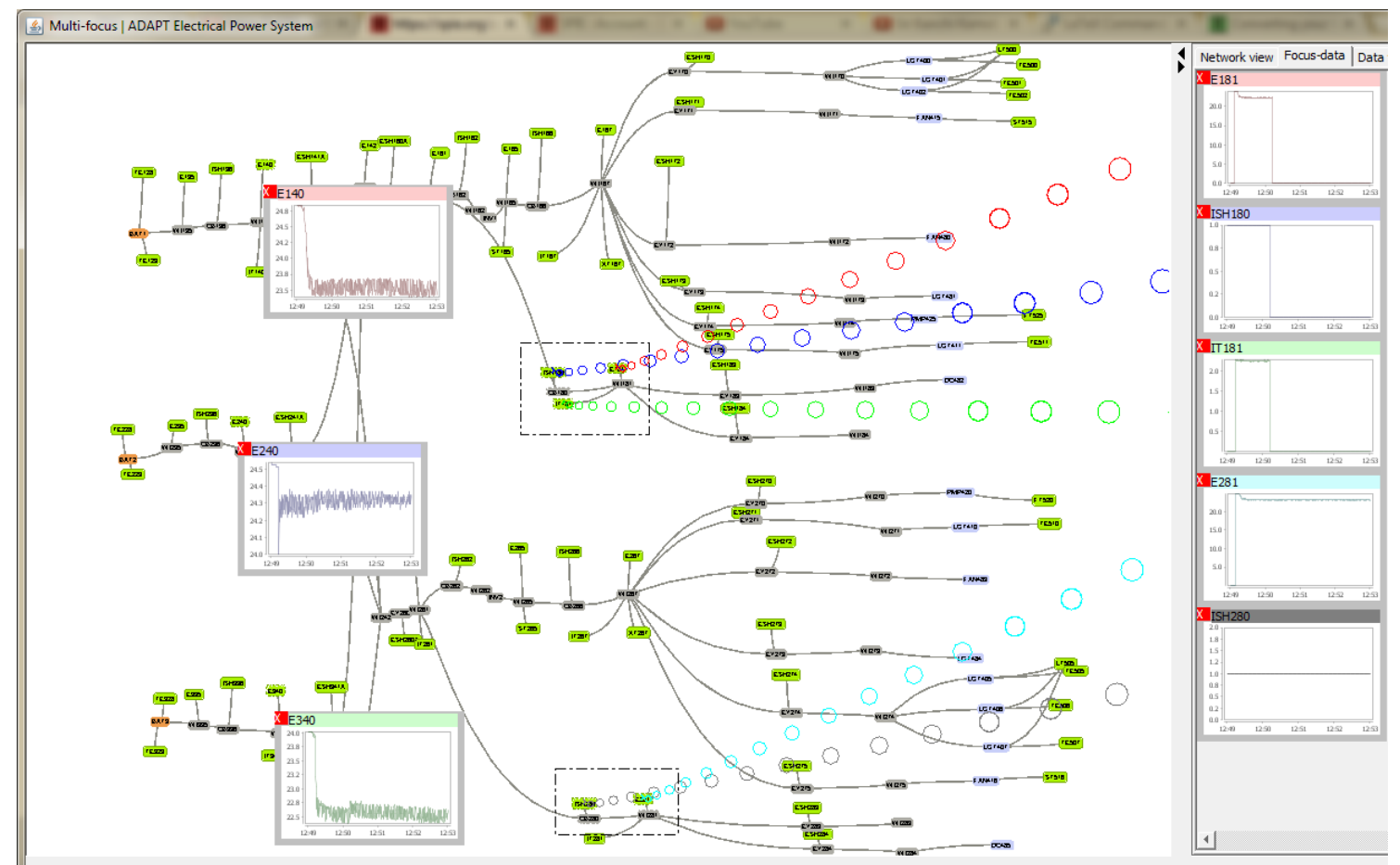

Figure 5: The time-series graphs of all the nodes inside the rectangular selections are aligned and shown in the side panel. Analysts can hover over the node to display the time-series graph as a tooltip. The graphs of the voltage sensors E140, E240 and E340, shown as floating windows, have been anchored in the network view by clicking on the network nodes.

(a) Medium distortion

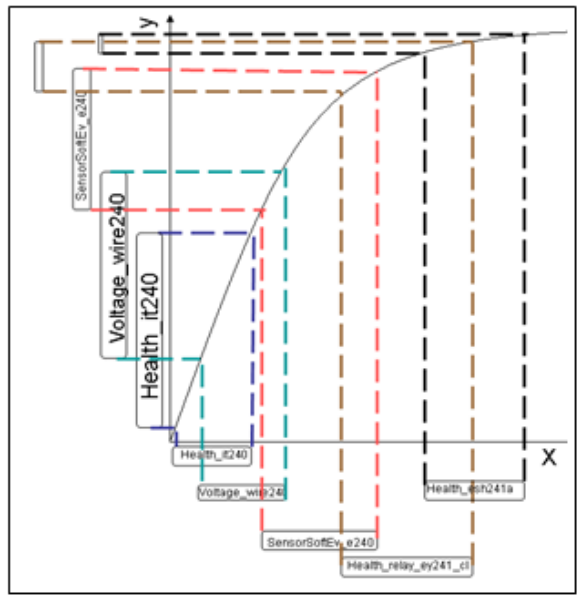

(b) High distortion

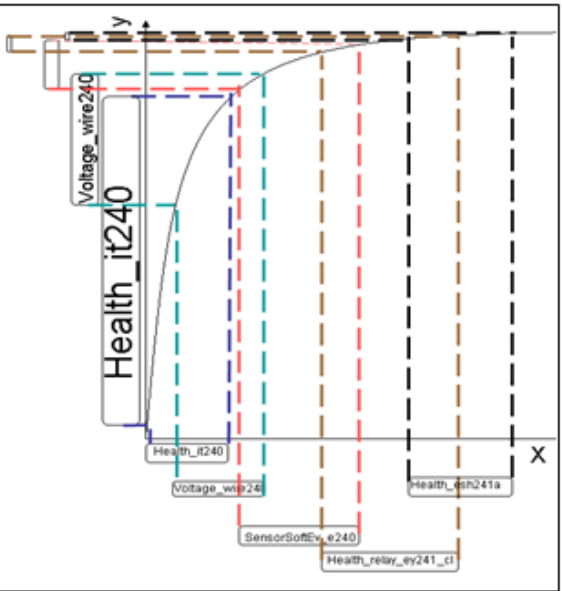

Figure 6: Arctan curve for different values of the distortion factor, $b$ and its effect on the node sizes: (a) $b=2.5$ (b) $b=5.0$. The original undistorted nodes are shown on the $x$-axis, while the nodes after distortion are shown in the $y$-axis. It is hard for an analyst to read the small labels along the $x$-axis, while the labels along the $y$-axis close to $(0,0)$ have become easier to read. 


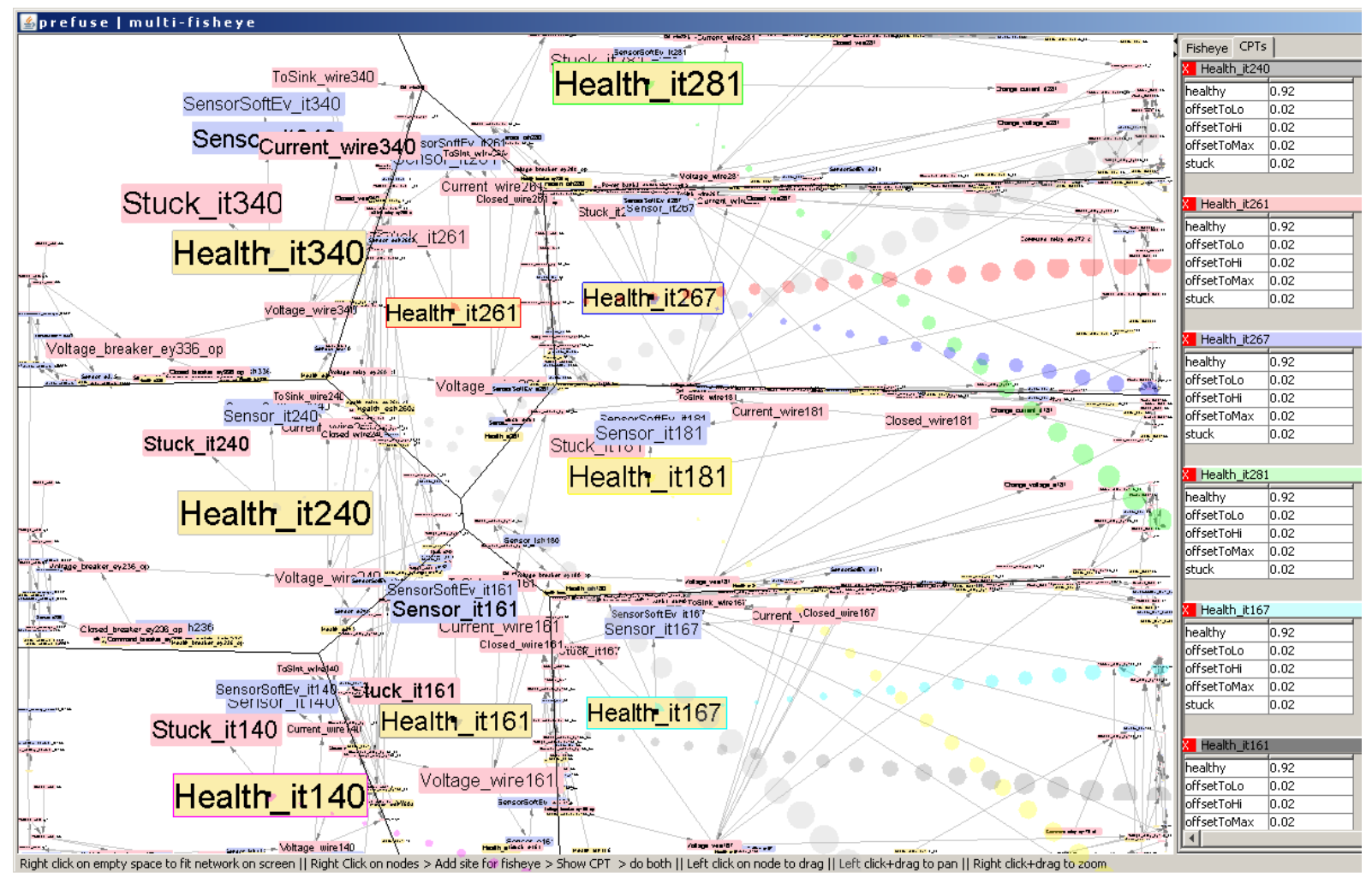

Figure 7: A zoomed-in visualization of nine focus nodes using the distortion factor $b=18$. The health node labels are all clearly readable. The node Health_it281 has a green color border; the bubbles connecting the node and the title bar of the detail window in the side panel are also represented in green color.

$$
r=a * \arctan \left(b * d_{\text {norm }}\right) * D_{\text {max }}
$$

where $a=\frac{1}{\arctan (b)}$ so that $r \in[0,1]$ before de-normalization.

Distortion can be increased or decreased by respectively increasing or decreasing the value of the distortion factor, $b$. We use the arctan function to distort; see Figure 6 for example arctan curves and their effects on the sizes of nodes including labels for different values of $b$. The topology or the relative position of the nodes are retained after the distortion (DG2).

Conversion from Polar to Cartesian co-ordinates is done using:

$$
\begin{aligned}
& x^{\prime}=r \cos (\theta)+x^{*} \\
& y^{\prime}=r \sin (\theta)+y^{*} .
\end{aligned}
$$

The above steps are applied to both the start and end coordinates of the node to get the transformed coordinates $\left(x_{s}^{\prime}, y_{s}^{\prime}\right)$ and $\left(x_{e}^{\prime}, y_{e}^{\prime}\right)$. Size distortion is then done by finding the new label based on the difference between the transformed coordinates $\left(x_{e}^{\prime}-x_{s}^{\prime}\right)$, where $x_{e}^{\prime} \geq x_{s}^{\prime}$. The new center position $\left(x_{c}^{\prime}, y_{c}^{\prime}\right)$ of the node is calculated as:

$$
\begin{aligned}
& x_{c}^{\prime}=\left(x_{e}^{\prime}-x_{s}^{\prime}\right) / 2 \\
& y_{c}^{\prime}=\left(y_{e}^{\prime}-y_{s}^{\prime}\right) / 2 .
\end{aligned}
$$

Figure 7 shows the multi-focus zooming effect on nine focused nodes for a distortion factor $b=18$ (DG1). The health node labels are all clearly readable (DG8). 


\section{APPLICATION}

Our novel multi-focus technique is implemented using the Prefuse framework. ${ }^{35}$ ADAPT Bayesian network as well as other networks have been visualized. Figure $9^{\dagger}$ shows the usage of the multi-focus technique in detecting faulty components in an electrical power system. Figure 10 shows the Munin2 Bayesian network with 1003 nodes where the children of a node (Proximal Myopathy) are zoomed using our multi-focus technique.

\subsection{Bayesian Networks}

The ADAPT Bayesian network, which can be used for automatic fault diagnosis, ${ }^{2}$ models an electrical power network that is representative of those found in aerospace vehicles. ADAPT has capabilities for power storage, distribution, and consumption, and contains batteries, electromechanical relays, circuit breakers, and different kinds of loads, such as pumps, fans, and light bulbs. Each component in ADAPT is modeled as a set of nodes. Health nodes (H) and the evidence (e) are of particular interest. The health nodes serve as the query variables, e.g. whether a component is defective or not, and the evidence nodes serve as the input variables, e.g. a command such as closing a relay to allow current from the battery to flow to the load. As an example fault scenario, ${ }^{2}$ suppose that $\mathbf{e}=\{$ CommandRelay $=$ cmdClose, SensorCurrent $=$ readCurrentLo, SensorVoltage $=$ readVoltageHi, SensorTouchSensor $=$ readClosed $\}$. This gives $\arg \max P(\mathbf{H} \mid \mathbf{e})=\{$ HealthBattery $=$ healthy, HealthLoad $=$ healthy, HealthCurrent $=$ stuckCurrentLo, HealthVoltage $=$ healthy $\}.$ In this scenario, given the evidence of the command and sensor readings, the current flows from the battery to the load as both of them are healthy, also the voltage sensor is healthy. So the defective component is the current sensor which reads low instead of high.

A visualization of the ADAPT network in which labels are hard to read (and with no distortion) is shown in Figure 1a. The network consists of 671 nodes and 790 edges. The font size of the labels is the same for all the nodes. With the network fit to screen, as it is here, it is impossible to read these labels on the computer screen making it extremely difficult to understand, validate or edit the Bayesian network. Using our software, the analyst interacts with the graph by changing the position of focus. The focus nodes that have been zoomed, will use a larger font for labels than their neighboring nodes as shown in Figure 7. It is now possible to read the node labels for the focus nodes and the nodes close to them. In general, multi-focus selection is used to make the labels readable and for comparing various nodes to explore their differences and similarities. The side panel in Figure 7 shows detailed information about the nodes, specifically the conditional probability tables, for further comparison and analysis. ${ }^{33}$ The bubbles help the user to trace the detail window to its corresponding node in the network view.

\subsection{Analytical Tasks}

Several problem solving tasks can be performed with a Bayesian network; we consider the validation and the diagnosis tasks.

\subsubsection{Validation Task}

The ADAPT Bayesian network is used for identifying failed components in an electrical power system. When validating such a network, the user may want to compare the CPTs of a set of nodes, for example, health nodes that represent health of different components but with similar conditional probability tables as shown in Figure 7. We investigate $P\left(H_{i} \mid p a\left(H_{i}\right)\right)$ where $H_{i} \in \mathbf{H}$ is a Bayesian network health node and $p a\left(H_{i}\right)$ denote the parent nodes of $H_{i}$. There can be multiple nodes that may be the major causal nodes for certain hidden or observable effects. These nodes can lie quite far-apart in a large Bayesian network as shown in Figure 8. Using our multi-focus and multi-window technique, they can be zoomed to analyze their CPTs.

\subsubsection{Diagnosis Task}

The diagnosis task investigates $P\left(H_{i} \mid \mathbf{e}\right)$ where $H_{i}$ is a Bayesian network health node and $\mathbf{e}$ is the evidence. Here, multi-focus can help a Bayesian analyst by allowing him or her to focus, at the same time, on multiple $H_{i}$ 's with interesting posteriors $P\left(H_{i} \mid \mathbf{e}\right)$. For example, it might be that multiple nodes have high posterior probabilities of being defective in a diagnostic Bayesian network such as ADAPT. If the nodes are distant and their labels are hard to read in the original network layout (as they can easily be in ADAPT and other Bayesian networks), our multi-focus technique provides valuable support for interactive analysis.

\footnotetext{
†http: / / www . youtube.com/watch?v=oJh1 kbQVcXc
} 


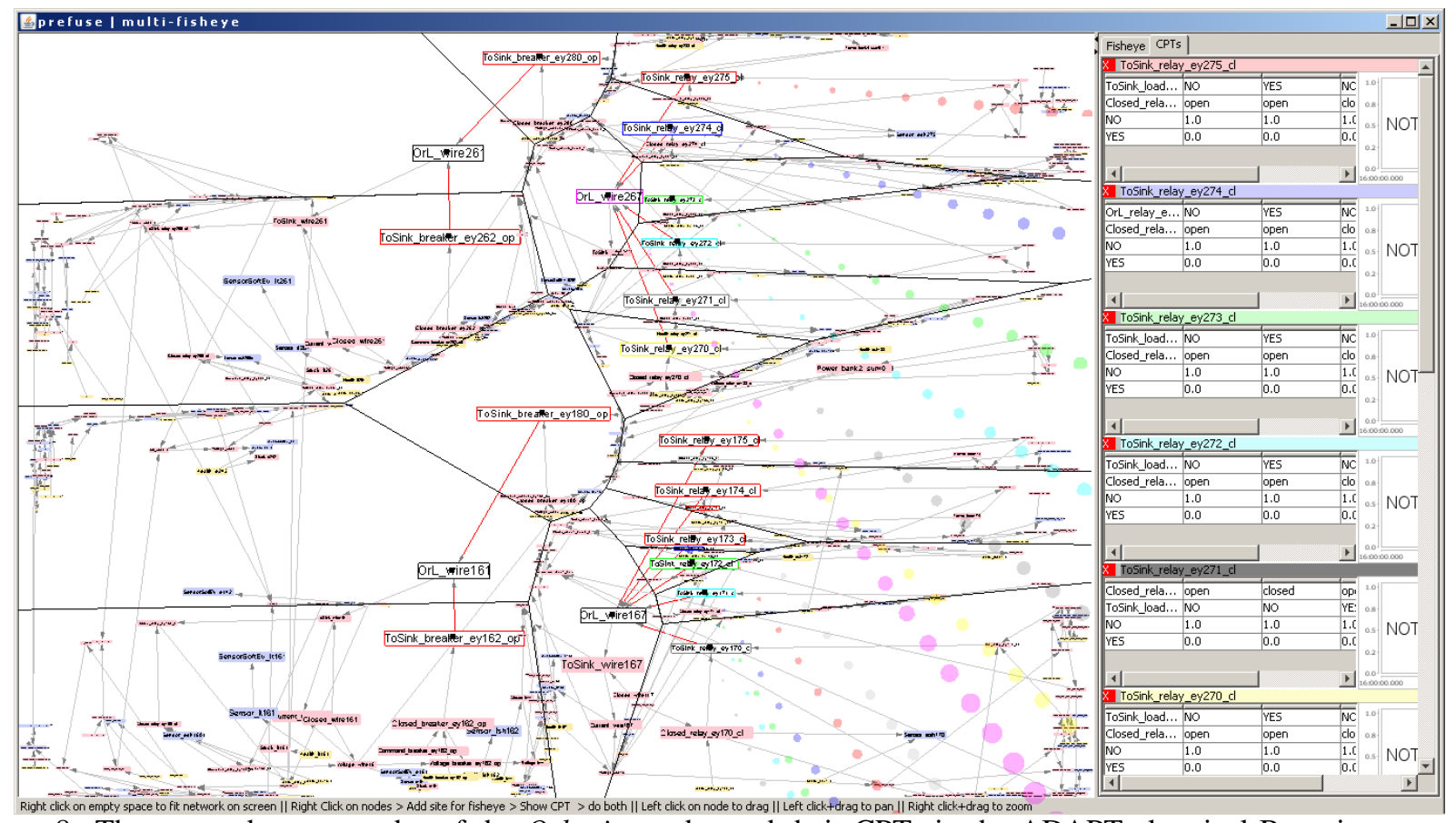

Figure 8: The zoomed parent nodes of the Orl_wire nodes and their CPTs in the ADAPT electrical Bayesian network. These zoomed node labels are readable on the computer screen even though they are difficult to read in this screenshot.

\subsection{Evaluation}

Our evaluation approach has been to explore complex Bayesian networks while taking note of how the tool aided an analyst in finding and remembering nodes of value during a problem solving session. Our exploration included one of the authors, Ole. He is a Bayesian network expert and has also worked with other Bayesian network visualization tools like Hugin, ${ }^{3}$ Netica $^{36}$ and GeNIe/SMILE. ${ }^{4}$ Many features and algorithms were explored, including alternative distortion approaches under rectangular and Voronoi polygonal partitioning. We ended up using user controllable distortion and Voronoi polygonal partitioning. Simplifying the controls and amplifying the mechanisms for remembering where one is in the network exploration process, were necessary for the user to make sense of the network.

The interface is a dramatic simplification over pop-up style controls, and helps focusing action on the essential gather and prune activities of a network visualization system. The interface felt agile and powerful to our Bayesian network expert as he was able to reformulate network questions several times a minute. He commented on and enjoyed discovering six mechanisms to orient, annotate and understand the relations between nodes. (1) Partitioning allowed him to quarantine (he used the word "sacred") parts of the network that contained interesting nodes. (2) The fisheye, he said, allowed him to highlight and remember which nodes he deemed important in a very visible way. (3) The bubbles gave him easy to follow indications of where important nodes were. (4) The motion of panning made the bubble lines show how distant the nodes were separate from other mechanisms. (5) Panning motions and mouse-over helped resolve nodes that were overlapping. (6) The use of node colouring helped to focus on nodes of similar types.

The interesting nodes in the network were found by using the search techniques as discussed in Section 3.1. For Bayesian networks, in reviewing the conditional probability tables associated with the focus nodes, our network expert found himself using a collect-review-dispense loop to home in on the conditional probability tables that needed to be compared. Often he would collect 10-20 of these tables and then prune down to 4-5 in one iteration of this collectreview-dispense loop. He described the activity as a network review, similar to code review in software engineering, as he poked around hunting and foraging with the support of the system's many memory aids. In particular, the tool helped in identifying important nodes for further analysis and comparison in the side panel. 


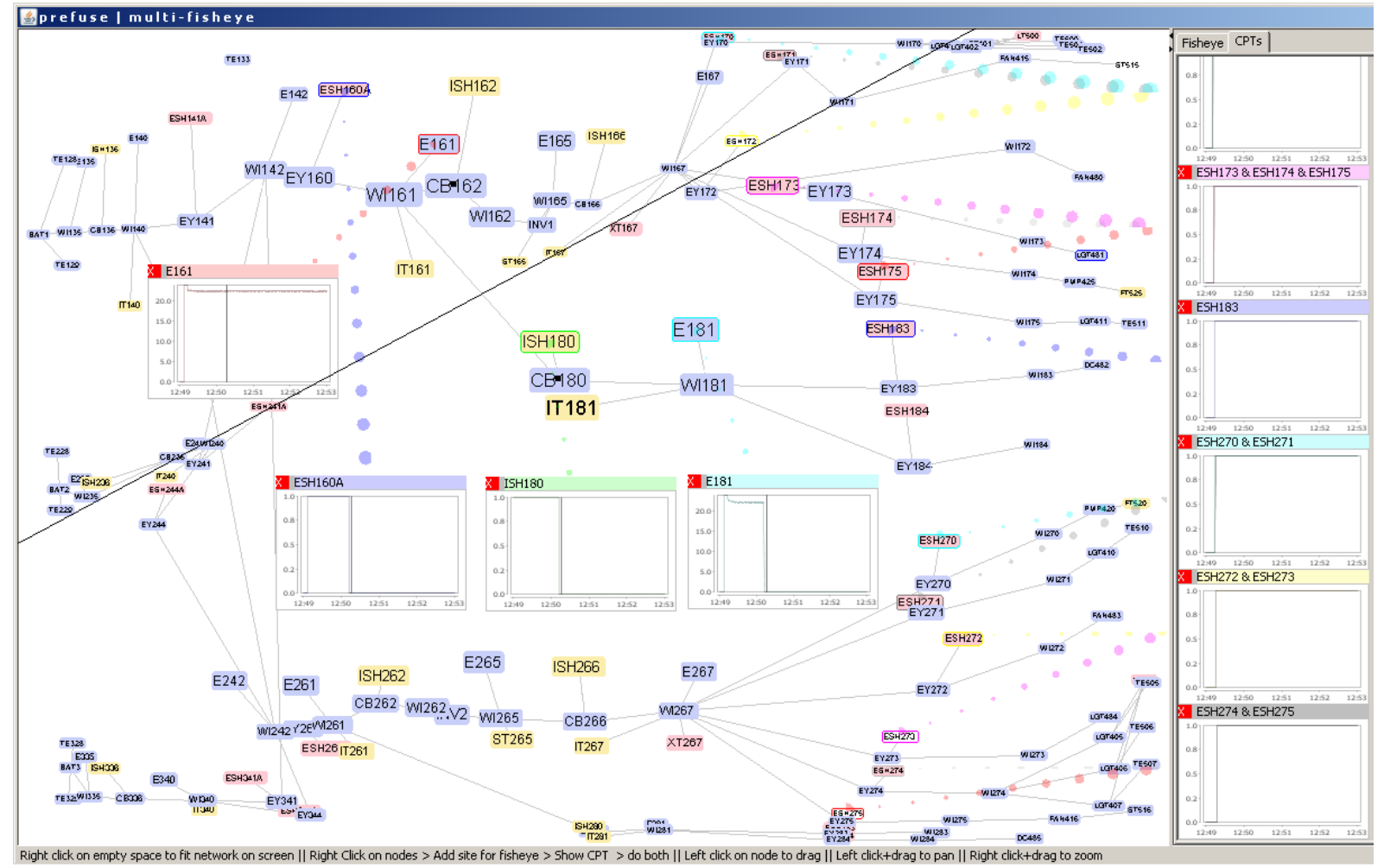

Figure 9: Time-series graphs of the zoomed sensor nodes in the ADAPT electrical power network. The node labels and their time-series graphs are readable for further analysis. The time-series graphs around the node CB180 show a drop in their reading, suggesting that the component CB180 is faulty.

\section{DISCUSSION AND FUTURE WORK}

Distortion such as fisheye views can increase the ability to keep context visible. Multi-focus approaches improve visual analysis by allowing comparison of different parts of a network, providing analysts with a collect-review-dispense analytical task scenario. Our system generates distortion boundaries which can reduce global distortion, reducing visual comparison challenges. In particular, our technique allows an analyst to interactively bring important parts of a network 'forward' by selectively zooming in, to be compared both structurally in the network and in a multi-window semantic display. ${ }^{33}$ Our system can be used to create over a dozen focus partitions.

This system gives simultaneous multi-focus and multi-window zooming capability that enables improved interactive visual exploration of Bayesian networks. In case of a failure in an electrical circuit, the user may want to find the faulty component. For this, a deeper analysis of each of the component is required. Using the multi-focus technique, similar components like voltage or current sensors, can be zoomed; using the multi-window technique their internal readings can be studied. The multiple windows which contain detailed data can be floating or aggregated in side panel. The side panel is designed to align and compare internal readings of multiple selected nodes. If there is any sudden drop or rise in the readings in any of the components, then it can be diagnosed further.

The multi-focus with the multi-window views shown in this paper promises to improve completion accuracy in network analysis; we hope to validate this in future work. We have used our technique in electrical networks (Figure 9), Bayesian networks (Figure 10) and even on social networks. This paper pushes for adding techniques to the arsenal of ways to allow users to better view large networks and analyze their complex problems. Analytics and reasoning are being done on increasingly complex datasets. This paper demonstrates improvements towards and calls for future work on systems that integrates scalable user interactivity into comparing parts and internal semantics of large-scale networks. 


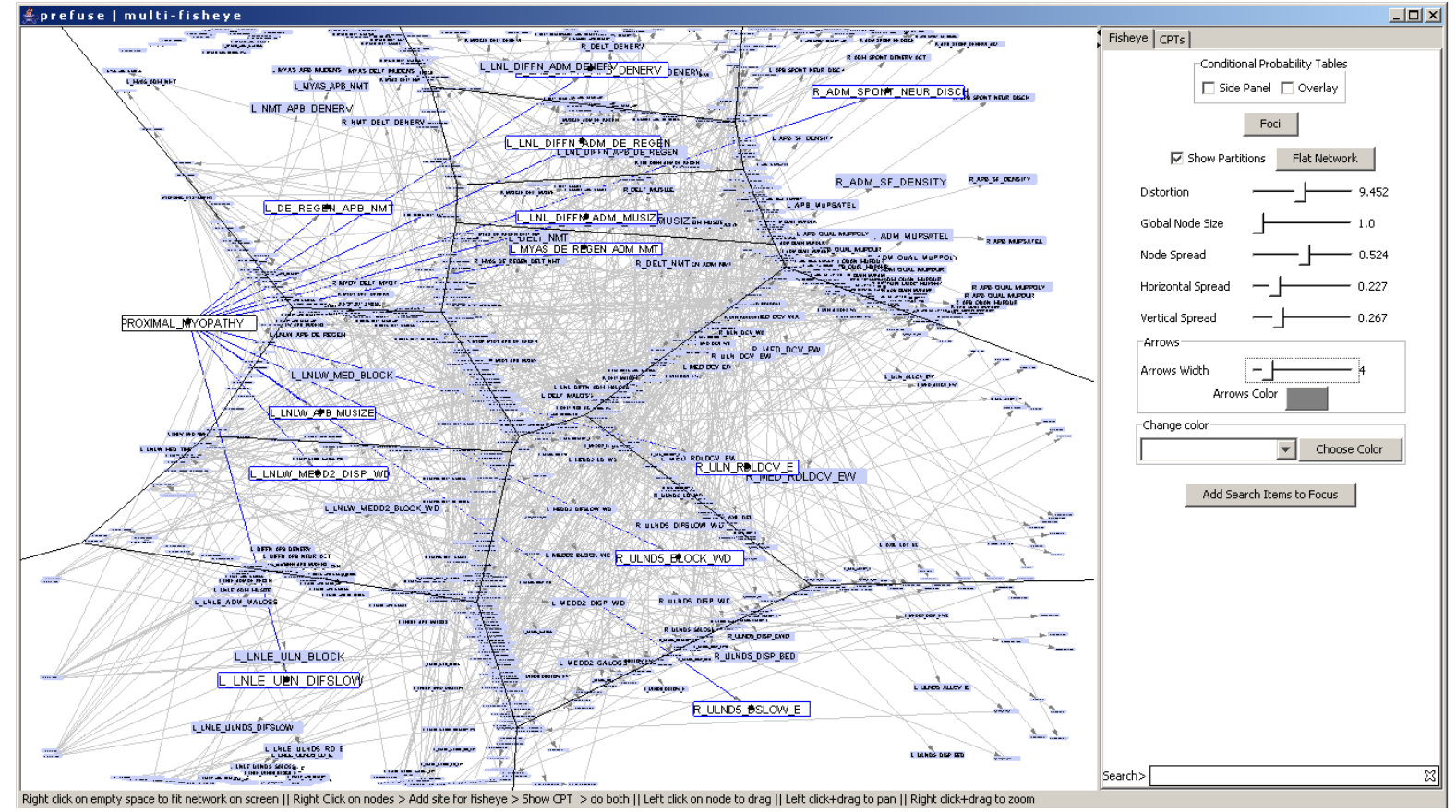

Figure 10: The large Munin2 Bayesian network and application of multi-focus on the children nodes ( $L \_L N L E \_U L N \_D I F L O W, L \_L N L W \_M E D 2 \_D I S P \_W O$ etc.) for a neural disorder disease node called Proximal Myopathy (the left most focus node). After the application of multi-focus zooming, the children and the disease nodes are clearly readable on the computer screen even though they are difficult to read in this screenshot.

\section{ACKNOWLEDGMENTS}

This material is based, in part, upon work supported by NSF grants CCF-0937044 and ECCS-0931978.

\section{REFERENCES}

[1] J.Pearl, [Probabilistic Reasoning in Intelligent Systems: Networks of Plausible Inference], Morgan Kaufmann (1988).

[2] Mengshoel, O. J., Chavira, M., Cascio, K., Poll, S., Darwiche, A., and Uckun, S., "Probabilistic Model-Based Diagnosis: An Electrical Power System Case Study," IEEE Trans. on Systems, Man, and Cybernetics (5) (2010).

[3] Andersen, S. K., Olesen, K. G., Jensen, F. V., and Jensen, F., "HUGIN - A Shell for Building Bayesian Belief Universes for Expert Systems," in [Proc. of IJCAI'89], 1080-1085 (Aug 1989).

[4] Druzdzel, M. J., "SMILE: Structural Modeling, Inference, and Learning Engine and GeNIe: A Development Environment for Graphical Decision-Theoretic Models," in [Proc. of AAAI'99], 902-903 (Jul 1999).

[5] Furnas, G. W., “Generalized fisheye views,” SIGCHI 17 (April 1986).

[6] Reinhard, T., Meier, S., and Glinz, M., “An improved fisheye zoom algorithm for visualizing and editing hierarchical models," in [Workshop on Requirements Engineering Visualization], REV '07 (2007).

[7] Storey, M. A. D., Fracchia, F., and Müller, H., "Customizing a Fisheye View Algorithm to Preserve the Mental Map," Journal of Visual Languages and Computing (10), 245-267 (1999).

[8] Carpendale, M. S. T., Cowperthwaite, D. J., and Fracchia, F. D., "Extending distortion viewing from 2d to 3d," IEEE Comput. Graph. Appl. 17, 42-51 (July 1997).

[9] Shneiderman, B., "The eyes have it: A task by data type taxonomy for information visualizations," in [Proceedings of the 1996 IEEE Symposium on Visual Languages ], 336-, IEEE Computer Society, Washington, DC, USA (1996).

[10] Fortune, S., "A sweepline algorithm for voronoi diagrams," in [Proceedings of the second annual symposium on Computational geometry], SCG '86, ACM, New York, NY, USA (1986).

[11] Cockburn, A., Karlson, A., and Bederson, B. B., "A review of overview+detail, zooming, and focus+context interfaces," ACM Comput. Surv. 41, 2:1-2:31 (Jan. 2009). 
[12] Sarkar, M. and Brown, M. H., "Graphical fisheye views of graphs," in [Proceedings of the SIGCHI conference on Human factors in computing systems ], CHI '92, 83-91, ACM, New York, NY, USA (1992).

[13] Lamping, J., Rao, R., and Pirolli, P., "A focus+context technique based on hyperbolic geometry for visualizing large hierarchies," in [Proc. SIGCHI Human factors in computing systems ], CHI '95 (1995).

[14] Sarkar, M., Snibbe, S. S., Tversky, O. J., and Reiss, S. P., "Stretching the rubber sheet: a metaphor for viewing large layouts on small screens," in [Proceedings of the 6th annual ACM symposium on User interface software and technology], UIST '93, 81-91, ACM, New York, NY, USA (1993).

[15] Formella, A. and Keller, J., "Generalized fisheye views of graphs," in [Proceedings of the Symposium on Graph Drawing ], GD '95, 242-253, Springer-Verlag, London, UK (1996).

[16] Gansner, E. R., Koren, Y., and North, S. C., "Topological fisheye views for visualizing large graphs," IEEE Transactions on Visualization and Computer Graphics 11, 457-468 (July 2005).

[17] Ghani, S., Riche, N., and Elmqvist, N., "Dynamic insets for context-aware graph navigation," in [Computer Graphics Forum ], 30(3), 861-870, Wiley Online Library (2011).

[18] Lee, T., "Coherent time-varying graph drawing with multifocus+ context interaction," IEEE TRANSACTIONS ON VISUALIZATION AND COMPUTER GRAPHICS 18(8) (2012).

[19] Bartram, L., Ho, A., Dill, J., and Henigman, F., "The continuous zoom: a constrained fisheye technique for viewing and navigating large information spaces," in [Proc ACM symposium on User interface and software technology], UIST '95, ACM, New York, NY, USA (1995).

[20] Schaffer, D., Zuo, Z., Greenberg, S., Bartram, L., Dill, J., Dubs, S., and Roseman, M., "Navigating hierarchically clustered networks through fisheye and full-zoom methods," ACM Trans. Comput.-Hum. Interact. 3 (1996).

[21] Plaisant, C., Grosjean, J., and Bederson, B., "Spacetree: supporting exploration in large node link tree, design evolution and empirical evaluation," in [Information Visualization, 2002. INFOVIS 2002. IEEE Symposium on], (2002).

[22] Munzner, T., Guimbretière, F., Tasiran, S., Zhang, L., and Zhou, Y., "TreeJuxtaposer: scalable tree comparison using Focus+Context with guaranteed visibility," ACM Trans. Graph. 22, 453-462 (July 2003).

[23] Tu, Y. and Shen, H.-W., "Balloon focus: a seamless multi-focus+context method for treemaps," IEEE Transactions on Visualization and Computer Graphics 14, 1157-1164 (November 2008).

[24] Batagelj, V., Didimo, W., Liotta, G., Palladino, P., and Patrignani, M., "Visual analysis of large graphs using (x,y)clustering and hybrid visualizations," in [Pacific Visualization Symposium (PacificVis), 2010 IEEE], (march 2010).

[25] Elmqvist, N., Riche, Y., Henry-Riche, N., and Fekete, J.-D., "Melange: Space folding for visual exploration," Visualization and Computer Graphics, IEEE Transactions on 16, $468-483$ (may-june 2010).

[26] Keahey, T. A., Gucht, D. V., Keahey, T. A., Jerde, N. G., and Keahey, T. E., "Nonlinear magnification," tech. rep., transformations,Proceedings of the IEEE Symposium on Information Visualization, IEEE Visualization (1997).

[27] Carpendale, M. S. T., Sheelagh, M., Carpendale, T., Cowperthwaite, D. J., and Fracchia, F. D., "3-dimensional pliable surfaces: For the effective presentation of visual information," in [In Proc. of UIST'95], ACM (1995).

[28] Kaugars, K., Reinfelds, J., and Brazma, A., "A simple algorithm for drawing large graphs on small screens," in [Proc. of the DIMACS International Workshop on Graph Drawing ], GD '94, Springer-Verlag, London, UK (1995).

[29] Herman, I., Melancon, G., and Marshall, M. S., "Graph visualization and navigation in information visualization: A survey," IEEE Transcations on Visualization and Computer Graphics 6(1), 24-43 (2000).

[30] Aurenhammer, F., "Voronoi diagrams a survey of a fundamental geometric data structure," ACM Comput. Surv. 23, 345-405 (September 1991).

[31] Sundararajan, P. K., Mengshoel, O., and Selker, T., "Multi-fisheye for interactive visualization of large graphs," in [The AAAI-11 Workshop on Scalable Integration of Analytics and Visualization], (2011).

[32] Balzer, M., Deussen, O., and Lewerentz, C., "Voronoi treemaps for the visualization of software metrics," in [Proceedings of the 2005 ACM symposium on Software visualization ], SoftVis '05, ACM, New York, NY, USA (2005).

[33] Cossalter, M., Mengshoel, O. J., and Selker, T., "Visualizing and understanding large-scale Bayesian networks," in [The AAAI-11 Workshop on Scalable Integration of Analytics and Visualization ], 12-21 (2011).

[34] Furnas, G. W., "A fisheye follow-up: further reflections on focus + context," in [Proceedings of the SIGCHI conference on Human Factors in computing systems ], CHI '06, 999-1008, ACM, New York, NY, USA (2006).

[35] Heer, J., Card, S. K., and Landay, J. A., "Prefuse: a toolkit for interactive information visualization," in [Proceedings of the SIGCHI conference on Human factors in computing systems ], CHI '05, ACM, New York, NY, USA (2005).

[36] Netica, "by Norsys Software Corp.," (1998). 